\title{
Accidental ingestion of cylindrical batteries in children - interventional or conservative management?
}

\author{
Gabriela Paduraru', Andreea-Madalina Nichita ${ }^{3}$, Florin Filip², \\ Elena Tataranu², Smaranda Diaconescu ${ }^{3}$ \\ 1 "Grigore T. Popa" University of Medicine and Pharmacy, lasi, Romania \\ 2 "Sf. Ioan cel Nou" Hospital, Suceava, Romania \\ ${ }^{3}$ Pediatric Gastroenterology Clinical Department, "Sf. Maria" Clinical Emergency Children's \\ Hospital, lasi, Romania
}

\begin{abstract}
Ingestion of cylindrical batteries is a rare condition in children. There are no clear guidelines regarding the appropriate management of this condition. We present 2 such cases; one patient required endoscopic removal of the battery, while in the other one the battery passed spontaneously. We recommend that such cases be admitted for monitoring and treated on an individual basis, according to the clinical picture, X-ray data and the availability of pediatric emergency endoscopy.
\end{abstract}

Keywords: cylindrical battery, ingestion, child, upper endoscopy, serial X-ray

\section{INTRODUCTION}

Battery ingestion is a common event in children due to their widespread use for watches, remote controls, toys etc. Consequently, clinical guidelines regarding the management of theses cases have been introduced in practice (1). Ingestion of cylindrical batteries is less common, mainly due to the large size of them, which makes it more difficult to swallow. Treatment consists of either close follow- up by serial $\mathrm{X}$-ray images or removal by surgery or endoscopy in case of symptoms or increase risk of perforation. We present our recent experience with two such cases who were managed differently but with favorable results in both situation.

\section{Case 1}

A 5-year old boy was admitted following an incidental ingestion of a cylindrical battery from a remote TV control device. At presentation, he was in moderate distress, complaining of nausea and mild retrosternal pain. There was no fever or vomiting. The physical examination showed a well-nourished boy with stable vital parameters, soft abdomen on palpation and no signs of oral bleeding. The plain abdominal X-ray taken in ER showed a cylindrical foreign body located in the upper abdomen, corresponding to the gastric area (Fig. 1), with the appearance suggestive for a cylindrical metal foreign body (battery).

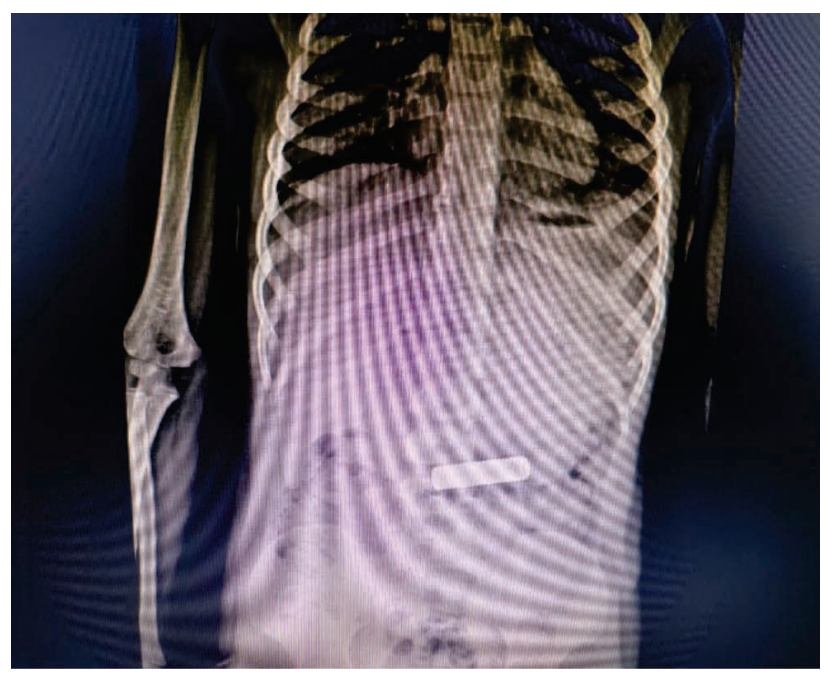

FIGURE 1. Antero-posterior X-ray showing a cylindrical battery lodged in the upper abdomen 
The patient was admitted on the Pediatric clinical department and placed on liquid diet and oral analgesics. During the next 24 hours his condition remained stable, with mild abdominal pain and discomfort, but no vomiting. A second plain abdominal X-ray was taken, which showed the battery in the same position as the previous one. Given the imaging result and the clinical condition of the patient, the endoscopic extraction of the battery was discussed, but in the tertiary proximity center there was no pediatric gastroenterologist on call. The patient was monitored and after 48 hours from the battery ingestion the general condition improved significantly, with no more pain or discomfort and regular diet tolerance. Another plain abdominal X-ray was taken and the battery was identified in the lower abdomen, probably the rectal area (Fig. 2). In the same day the patient spontaneously eliminated the battery, which was found to be intact (Fig. 3). He had no pain, bleeding or other symptoms and was discharged the same day with the indication of careful monitoring to avoid possible future ingestions.

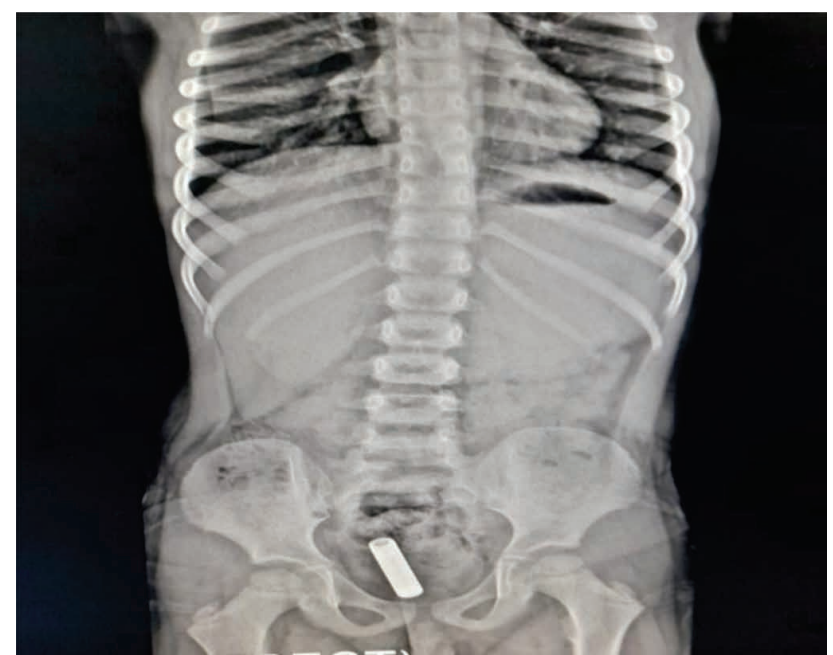

FIGURE 2. Antero-posterior X-ray showing cylindrical battery lodged in the lower abdomen

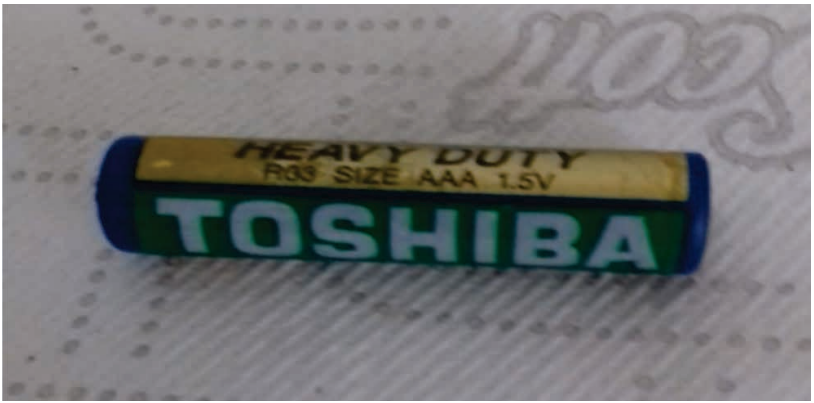

FIGURE 3. Intact cylindrical battery eliminated

\section{Case 2}

A 5 years old female was admitted to the department of pediatric gastroenterology following the ingestion of a foreign body, which her parents referred to as a cylindrical battery. The patient had no complaints on admission and clinical examination revelead no signs of immediate airway compromise or abdominal distress. She underwent an anteroposterior plain chest and abdominal X-ray (Fig. 4), confirming the presence of a foreign body (elongated radio opaque shadow) at the level of L2-L3 vertebras, in the lower part of the stomach. There was no evidence of obstruction or perforation. The esophagogastroscopy provided visualization of a foreign body - a cylindrical battery (Fig. 6), placed between the folds of the gastric mucosa, visible in the fundic region, subcardial, tangential to the cardia.The extraction was performed using a polypectomy snare (Fig. 5 and 6).

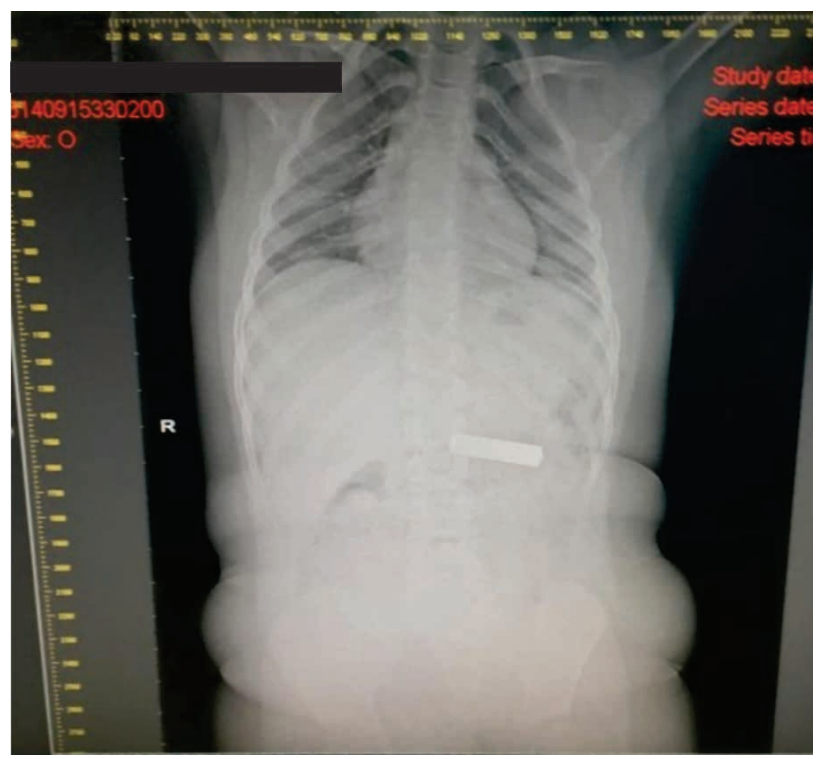

FIGURE 4. Anteroposterior X-ray showing a cylindrical battery lodged in the stomach

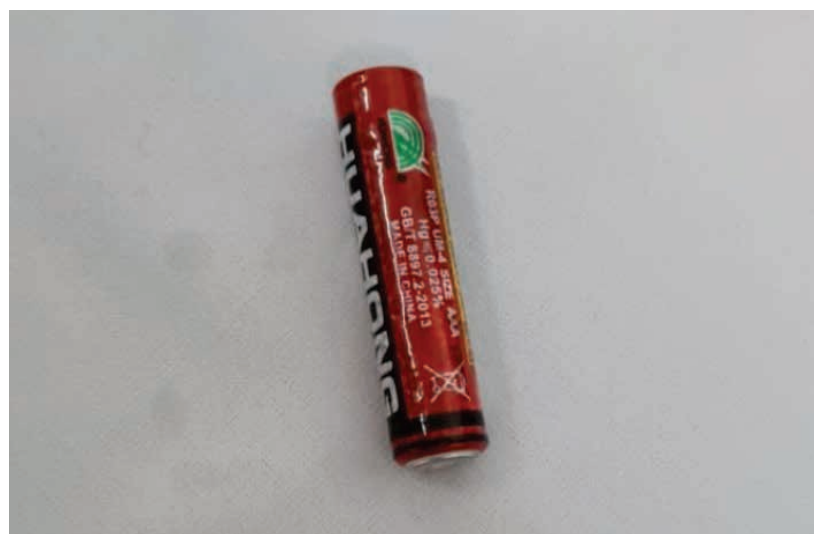

FIGURE 5. Cylindrical battery after endoscopic removal

\section{DISCUSSION}

Ingestion of batteries is well documented in the pediatric population, since it became quite frequent after the large scale use of button batteries. Button batteries are easy to swallow by a curios child or by accident (2). They can cause local necrosis and es- 


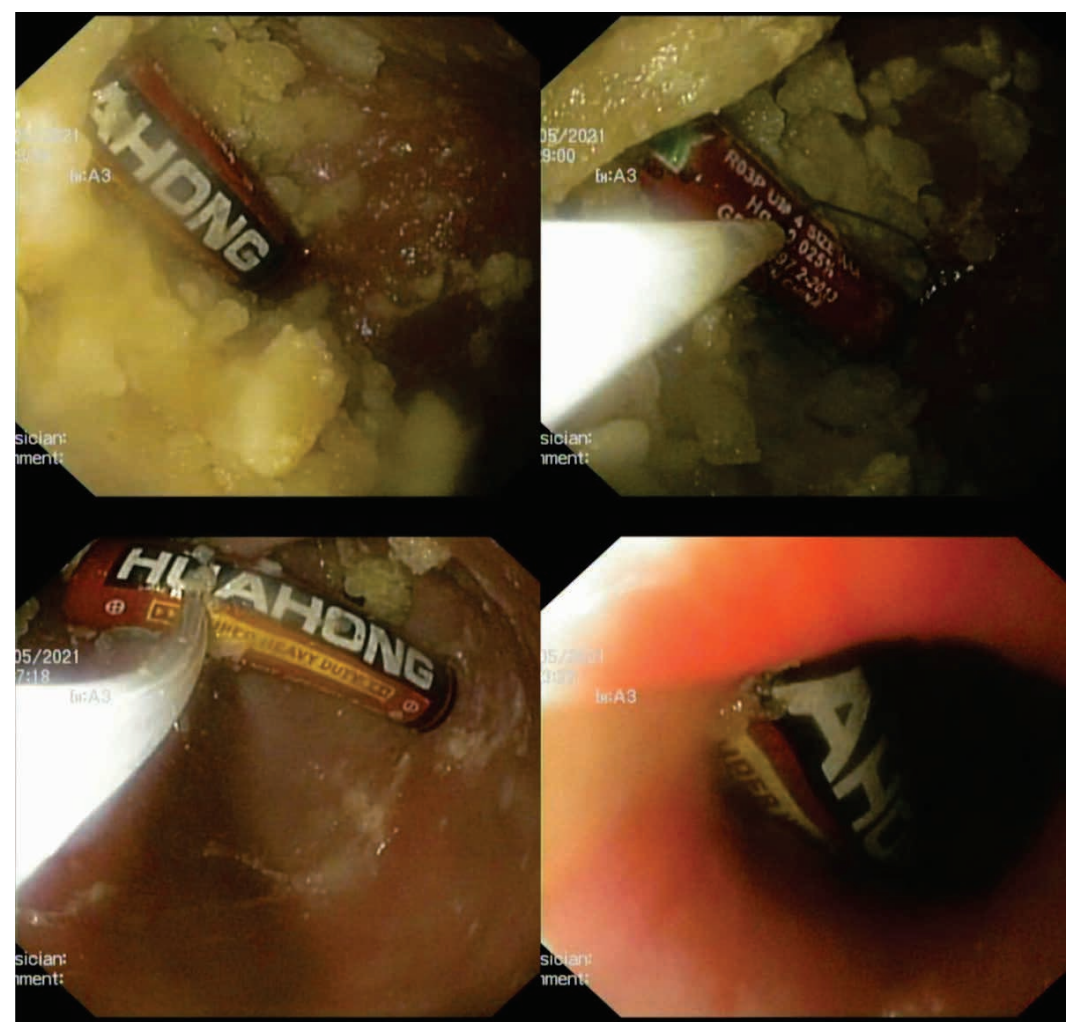

FIGURE 6. Endoscopic extraction of cylindrical battery

ophageal perforation, with potentially severe complications. Guidelines regarding the appropriate management of such events have been developed. It is highly recommended that batteries located in the esophagus be removed urgently (3). However, ingestion of cylindrical batteries is rare in practice given their larger size. It occurs mostly in people aged 6-39 years and some papers reported their ingestion especially in psychiatric patients or prison inmates $(4,5)$. Their ingestion has been related to corrosive and toxic damage usually occurring weeks, not days, after ingestion. Although rare, cases of mucosal damage after cylindrical battery ingestion appeared in less than 48 hours after the event were described $(6,7)$. The condition of the battery surface (damaged or intact) at the time of ingestion is important. Given the small number of cases reported in the literature, no guidelines related to their removal have been implemented. A paper reporting 125 cases of ingested batteries only found six situations in which cylindrical batteries were involved (8); in all of these cases, the batteries passed spontaneously through the gastrointestinal (GI) tract. No complications or the need to remove the batteries were reported. A comprehensive literature research involving ingestion of cylindrical batteries found that most cases were asymptomatic or presented with minor upper GI complaints or abdominal pain (9). However, there were many reports of cases requiring either endoscopic or surgical removal of cylindrical batteries, possibly related to adhesions or dysmotility preventing the passage of batteries (10-12).

There are no clear guidelines regarding the ingestion of cylindrical batteries. Given their length, which is usually $>2.5 \mathrm{~cm}$, they may become entrapped in the stomach or other parts the GI tract (9). Intact cylindrical batteries carry a low risk of caustic damage. Cylindrical cells are typically alkaline-manganese or nickel cadmium (rechargeable) and when ingested are capable of causing both corosive and toxic damage. Even in such conditions, given their large size and volume of toxic contents (alkaline batteries include zinc, manganese, mercury and lithium), cases of mucosal chemical damage and mercury poisoning were reported (2). Such damage would occur if the integrity of the battery surface was deliberately damaged (as in psychiatric patients) or as a result of sustained acid attack from gastric contents over a period (because of its size the battery could be stationary in the stomach and the passage through the pylor is delayed). Mucosal necrosis seems to be much less likely with cylindrical batteries that with the button variety. It would seem that major complications following cylindrical battery ingestion relate to the battery's loss of integrity. Clinical symptoms relating to such an event my be delayed, but are usually unequivocal and require prompt clinical response when they occur, generally surgical because it is to late for the endoscopic intervention.

In our paper we presented two cases of incidental ingestion of cylindrical batteries in 5 years old chil- 
dren. One child required endoscopic removal of the battery, the other one passed it spontaneously three days after ingestion. Both patients were carefully monitored during admission, and followed with serial plain X-rays of the abdomen. The patient who passed the battery spontaneously showed progression of the battery along the GI tract and had minor GIrelated symptoms. The second patient had no complain at admission or post foreign body extraction, probably due to the rapid endoscopic intervention, in less than 24 hours after ingestion.

\section{CONCLUSIONS}

Incidental ingestion of cylindrical batteries is rare in the pediatric population. Since there are no estab- lished guidelines regarding the management of these cases, the patients should be admitted for close monitoring and serial follow- up with plain abdomen Xrays. Final decision regarding removal of the battery (by either endoscopy or open surgery) or simple observation until spontaneous passage of the battery should be taken on an individual basis. The availability of the pediatric gastronterologist and the team (anesthesiologist, endoscopy assistant) as well as the time in which the intervention can be performed are essential in these cases.

Conflict of interest: none declared Financial support: none declared

\section{REFERENCES}

1. ASGE Standards of Practice Committee, Ikenberry SO, Jue TL, Anderson MA, et al. Management of ingested foreign bodies and food impactions. Gastrointest Endosc. 2011 Jun;73(6):1085-91.

2. Litovitz T, Schmitz BF. Ingestion of cylindrical and button batteries: an analysis of 2832 cases. Pediatrics. 1992 Apr;89(4Pt2):747-57.

3. David TJ, Ferguson AP. Management of children who have swallowed button batteries. Arch Dis Child. 1986 Apr;61(4):321-2.

4. Gitlin DF, Caplan JP, Rogers MP, et al. Foreign body ingestion in patients with personality disorders. Psychosomatics. 2007 Mar-Apr;48(2):162-6.

5. O' Sullivan ST, Reardon CM, McGreal GT, et al. Deliberate ingestion of foreign body by institutionalized psychiatric hospital patients and prison inmates. Ir J Med Sci. 1996 Oct-Dec;165(4):294-6.

6. Tien T, Tanwar S. Ingestion of cylindrical batteries and its management. BMJ Case Rep. 2017 Jan 17;2017:bcr2016218448.

7. Cyrany J, Melek J, Dedek P, Toms J, Rejchrt S. Cylindrical battery ingested by a 1-year-old baby - does the voltage matter? Endoscopy. 2014;46 Suppl 1 UCTN:E543-4.

8. Litovitz TL. Battery ingestion: product accessibility and clinical course. Pediatrics. 1985 Mar;75(3):469-76.

9. Hammami MB, Alkaade S, Piraka C, et al. Endoscopic retrieval vs observation in cylindrical battery ingestion. Ochsner Journal. 2019;19:157-65.

10. Young TL, Lubitz RM. Cylindrical battery ingestion: a case of endoscopic retrieval. J Tenn Med Assoc. 1989 Aug;82(8):415-6.

11. Kaplan G, Totten VY. Repeat cylindrical battery ingestion. Emerg Med. 1993 Dec;5 (4):257-60.

12. Lim JK, Kaltenbach TR, Sirinian E, et al. Endoscopic removal of two leaking alkaline batteries. Gastrointest Endoscop. 2006 Mar;63(3):497-98. 